\title{
Binary Diffing as a Network Alignment Problem via Belief Propagation
}

\author{
Elie Mengin \\ SAMM, EA 4543 \\ Université Paris 1 Panthéon-Sorbonne, Paris, France \\ Quarkslab SA \\ 13 rue Saint-Ambroise, Paris, France \\ elie.mengin@gmail.com
}

\author{
Fabrice Rossi \\ CEREMADE, CNRS, UMR 7534 \\ Université Paris-Dauphine, PSL University, Paris, France \\ fabrice.rossi@dauphine.psl.eu
}

\begin{abstract}
In this paper, we address the problem of finding a correspondence, or matching, between the functions of two programs in binary form, which is one of the most common task in binary diffing. We introduce a new formulation of this problem as a particular instance of a graph edit problem over the call graphs of the programs. In this formulation, the quality of a mapping is evaluated simultaneously with respect to both function content and call graph similarities. We show that this formulation is equivalent to a network alignment problem. We propose a solving strategy for this problem based on max-product belief propagation. Finally, we implement a prototype of our method, called QBinDiff, and propose an extensive evaluation which shows that our approach outperforms state of the art diffing tools.
\end{abstract}

Index Terms-Binary Diffing, Binary Program Analysis, Graph Edit Distance, Network Alignment, Belief Propagation

\section{INTRODUCTION}

Static program analysis is the process of analyzing and predicting the possible execution behaviors and outcomes of a program without actually executing it. It can be performed on the source code of the program or, with more difficulty, on the binary executable. Static program analysis has a wide variety of applications such as vulnerability detection, patch analysis, malware detection, software clone detection, etc.

In most of the cases, static analysis of binaries needs human expertise which is leveraged using specific software tools. Among those tools, differs are particularly useful as they allow the analyst to focus on the differences between a previously analyzed program and the one currently under investigation, enabling knowledge capitalization. Finding the differences between two programs in binary form only is known as the binary diffing problem.

Several different formulations of the problem have been given, mostly depending on the use case or the desired granularity but also implicitly induced by the solving approach [1]. In this paper, we address the problem of finding the best possible one-to-one correspondence between the respective functions of two programs in binary form.

Following previous authors [2], [3], [4], we leverage a graph edit formulation of binary diffing: we find an (almost) optimal transformation of the call graph of program $A$ into the call graph of program $B$, with respect to some specific edit costs.

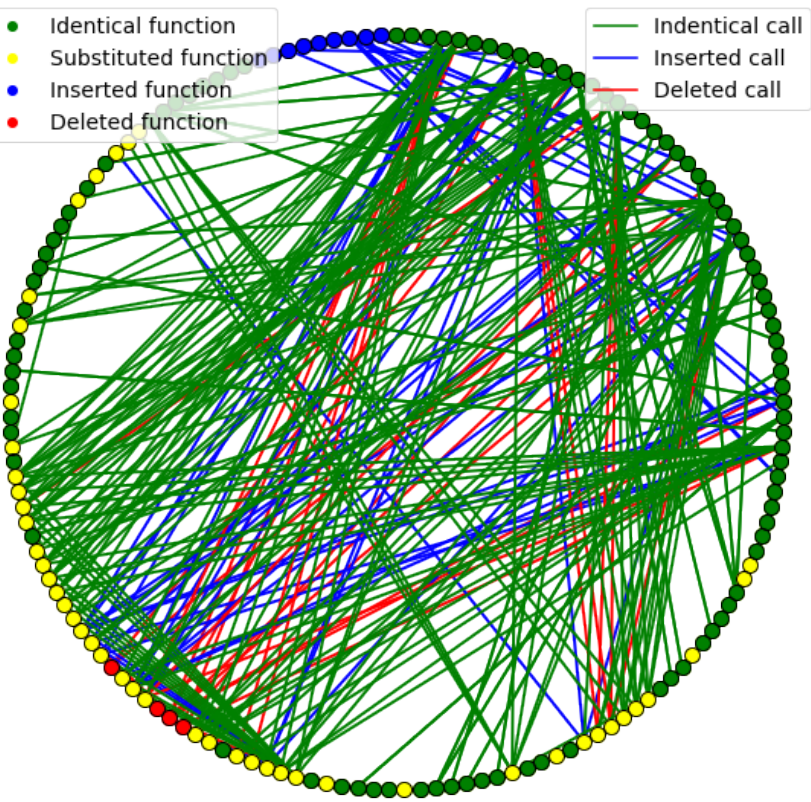

Fig. 1: Binary diffing as an alignment of call graphs. This layout represents the superposition of functions (dots) and function calls (lines) of two binaries (libz-1.2.4.3 vs libz1.2.6.1). Such mapping provides useful information to an analyst. In this figure, green dots and lines represent functions and calls that remained identical from a program to the other, and thus correspond to duplicated code. Blue (resp. red) elements represent inserted (resp. deleted) items, and may indicate added (resp. removed) functionalities. Yellow dots correspond to matched functions which content has been modified (substituted). They may thus record the functions that have been patched during the release. The figure highlights the interest of leveraging the function call consistency in order to find the best possible function correspondence. 
We then show that this formulation is equivalent to a network alignment problem. Following [5], we propose an efficient approximate solver of this problem based on max-product belief propagation. In summary, our contributions are:

- a new formulation of the binary diffing problem as a graph edit distance problem;

- an equivalent formulation as a network alignment problem;

- an efficient solver, QBinDiff, based on max-product belief propagation;

- a new diffing benchmark dataset consisting in more than 60 binaries and over 800 manually extracted ground truth correspondences;

- an extensive evaluation of our approach by comparing to other common matching methods, as well as other state of the art function similarity measures;

Our experimental results show that the proposed approach outperforms other diffing methods in almost all problem instances. Moreover, they highlight that function similarity measures originally designed for near-duplicate detection are not fitted to compute diffing assignments. Finally, they suggest that our problem formulation is particularly adapted to address the binary diffing problem.

The rest of paper is organized as follows. Section II introduces in more details the binary diffing problem and reviews some existing solutions. Our proposed formalization as an optimization problem is described in Section III. while Section IV] summarizes the maximization algorithm used to solve the problem. Section $\mathrm{V}$ is dedicated to the experimental evaluation of our solution.

\section{BINARY DIFFING}

\section{A. Graph representation and function matching}

In binary analysis, in order to consider its different potential execution behaviors, a binary executable can be represented as a directed attributed graph. In this graph, nodes stand for uninterrupted sequences of instructions, called basic blocks, and edges indicate the possible jumps from a basic block to another (conditional jumps, calls or returns). Such graph theoretically represents all possible execution paths of the program. It is known as the control-flow graph (CFG) [6].

Another common representation of a program consists in a partition of the control-flow graph according to the call procedures. The resulting directed attributed graph is composed of nodes denoting the different program functions and edges registering the calls among them. It is known as call graph (CG). This representation corresponds to a higher level of abstraction than the $\mathrm{CFG}$, closer to the developer point of view.

Note that retrieving both the CFG and the CG of a binary executable is a challenging problem that may not be solved exactly in some cases [6]. In this paper, we assume that both the CFG and CG can be reliably obtained from the executable programs.

In this paper, we define the binary diffing problem as the problem of matching call graphs. We want to match functions from one program to the functions of another such that they share similar functionalities (node content similarity) and they call other functions in a similar way (induced edge similarity). As a result, when a matching is satisfactory, the remaining differences between the call graphs can be interpreted as meaningful modifications from one program to the other.

As any binary diffing formulation, our definition requires a measure to assess the quality of a matching between two call graphs. This measure should evaluate the similarity of matched functions, as well as the relevance of the resulting graph alignment. Therefore, any binary diffing instance is characterized by the given function similarity and topology similarity measures. Once they are properly defined, we may formulate the binary diffing problem as an assignment problem which solution is the best one-to-one correspondence between the functions of both program.

In the rest of this section, we present a short state-of-the-art of existing methods to measure function similarity, as well as common proposed approaches to compute the best function mapping.

\section{B. Binary code similarity}

The problem of measuring how much two pieces of binary code are similar is a fundamental problem of program analysis. Indeed, two seemingly different binary executables may have the exact same functionality. Such programs are said to be semantically equivalent while syntactically different. Conversely, two slightly divergent pieces of code may have very different behaviors when executed. Moreover, syntactic similarity is relatively easy to compute but can lead to incorrect matching, while full semantic characterization is undecidable (and heuristics tend to be computationally expensive). Therefore, the definition of a similarity measure between two binary functions generally involves an arbitrary trade-off between syntactic [7], [8], [9] or semantic comparisons [10], [11], [12].

Many recent approaches propose a mixed strategy. The idea is to use simple syntactic features and to encode part of the function semantic through its control-flow graph. For instance, Gemini [13] introduces a Siamese graph neural network to learn the common features of two semantically similar functions. The model considers a very simple representation of the function instructions as well as the basic block layout of the CFG. It then embeds these features into a metric space where semantically similar functions are likely to have close coordinates. Once every function representation is projected into this metric space, pairwise similarity scores can be computed very efficiently using common vector-based distance computation routines.

Based on the same basis of Gemini, GraphMatching [14] proposes to enhance the model with an attention mechanism based on the structure of both function CFGs. However since it actively uses the topology of both graphs during the similarity score computation itself, GraphMatching can not benefit from fast vector-based distance computation as Gemini does. Therefore, the time required to compute all pairwise 
similarity scores may rise significantly with the size of the binaries.

Another alternative is Asm2Vec [15] which also provides vector representations for binary functions but in an unsupervised way that does not need matching pairs of functions. DeepBinDiff [16] improves over Asm2 Vec by working at the level of basic blocks. The embedding of a basic block is based on its content but also the one of its closer neighbors. It uses an adaptation of a graph embedding algorithm, text-attributed deep walk (TADW) [17] to extract a vector representation of each basic blocks among both binaries. To do so, it first merges the inter-procedural CFGs of both programs based on the binary symbols and then runs the TADW algorithm to compute the embedding of each basic blocks in this larger graph. Note that this approach is designed to proceed the diffing at a basic block granularity, whereas ours seeks a mapping between the functions of each binaries.

\section{Call graph matching}

Given a similarity measures between functions, one must now define a criteria of quality of the call graph alignment. In practice, such measure is closely related to the chosen function matching strategy.

The simplest solution for matching two call graphs consists in disregarding the call graph structure itself and simply looking for a one-to-one mapping that maximizes the sum of the similarities between the matched functions. In order to address the binary diffing problem, this would be the natural matching strategy used by methods originally designed to retrieve similar functions such as Gemini [13] or GraphMatching [14]. Finding the best match reduces to an instance of the linear assignment problem also know as the maximum weight matching problem (MWM). This is well known problem for which optimal solutions can be found exactly in polynomial time, using e.g. the Hungarian algorithm [18]. The major drawback of this approach is that the resulting mapping might be highly inconsistent with regards to the call graph structure of the two programs.

To overcome this issue, other approaches such as BinDiff [19] and DeepBindiff [16] propose instead to use matching algorithms designed to approximate the maximum common edge subgraph problem (MCS) [20]. Therefore, they implicitly define binary diffing as an instance of the MCS. This problem consists in finding the node correspondence which induces the maximum number of overlapping edges when aligning the graphs [21]. The general idea of those solutions is to expand in an iterative way the partial solution by seeking potential matches in the neighborhood of the current mapping (caller or callee of any already matched function). Though in practice this strategy proved to provide satisfying results, it suffers from a major limitation: by restricting new matches to belong to the respective neighbors of the current partial mapping, it prevents the assignment of potentially better nonlocal correspondences. Therefore, this strategy mostly consists in finding a locally-consistent mapping whereas a globally better assignment potentially exists.

\section{Graph edit distance}

A natural way to globally assess the quality of a matching is to consider it as a particular case of graph edition. One defines a set of graph edit operations on both nodes and edges of the graphs, and assigns to them a cost. The cost of a series of operations, also called an edit path, is simply the sum of the costs of said operations. Then, the edit path that transforms graph $A$ into $B$ at the minimum cost is called an optimal edit path and the resulting edit cost is known as the graph edit distance [22].

A matching can be viewed as a particular edit path in which matched nodes result from an edition, whereas unmatched nodes in $A$ are considered removed and unmatched nodes in $B$ inserted. The operation on the edges (insertion/deletion/edition) are then completely induced by those on the nodes (see Section III for details). Therefore, there is a close relationship between an optimal matching and an optimal edit path.

Unfortunately, the computation of the graph edit distance of two arbitrary graphs is known to be NP-complete and even APX-hard [23]. Though exact algorithms exist, they rapidly become intractable as the number of vertices rises [22]. In practice, the computation of the GED of graphs of more than a hundred nodes must be approximated. Note that the abovementioned MCS problem is also NP-complete.

Several approaches previously proposed to compare programs in binary form through a GED formulation [2], [3], [4]. However, in order to compute an approximated solution, all of them refer to Riesen and Bunke's linear programming relaxation [24], which reduces to a MWM formulation of the binary diffing problem with a function similarity measure taking into account the number of incident edge of each function.

In this paper, we propose to directly address the GED problem through an equivalent network alignment problem formulation. In this form, the globally optimal edit-path can be efficiently approximated by means of a message passing framework.

\section{FORMALIZATION}

The novelty of our approach lies in the reformulation of the graph edit distance calculation into a network alignment problem (NAP) which can then be solved (approximately) with a dedicated message passing algorithm. We give a formal derivation of the NAP in the present section.

\section{A. Binary diffing as a graph edit distance problem}

Let us consider two binary executables $A$ and $B$. We assume that adapted disassembly tools are used to represent them by their respective call graph $G_{A}=\left(V_{A}, E_{A}\right)$ and $G_{B}=\left(V_{B}, E_{B}\right)$. The vertices $V_{A}=\{1, \ldots, n\}$ and $V_{B}=$ $\left\{1^{\prime}, \ldots, m^{\prime}\right\}$ represent the functions of $A$ and $B$. The edges $E_{A}=\left\{(i, j) \mid i, j \in V_{A}^{2}, i \neq j\right\}$ and $E_{B}=\left\{\left(i^{\prime}, j^{\prime}\right) \mid i^{\prime}, j^{\prime} \in\right.$ $\left.V_{B}^{2}, i^{\prime} \neq j^{\prime}\right\}$ represent the function calls (e.g. $(i, j) \in E_{A}$ encodes the fact that function $i$ calls function $j$ in program $A$ ). Without loss of generality, self-loops (a.k.a. recursive calls) are 
TABLE I: Graph edit operations and respective costs.

\begin{tabular}{ll}
\hline Operation & Cost \\
\hline edit function & $c\left(i \rightarrow i^{\prime}\right)=d_{i i^{\prime}}$ \\
delete function & $c(i \rightarrow \epsilon)=d_{\epsilon}$ \\
insert function & $c\left(\epsilon \rightarrow i^{\prime}\right)=d_{\epsilon}$ \\
\hline edit call & $c\left((i, j) \rightarrow\left(i^{\prime}, j^{\prime}\right)\right)=d_{i i^{\prime} j j^{\prime}}$ \\
delete call & $c((i, j) \rightarrow \epsilon)=d_{\epsilon \epsilon}$ \\
insert call & $c\left(\epsilon \rightarrow\left(i^{\prime}, j^{\prime}\right)\right)=d_{\epsilon \epsilon}$ \\
\hline
\end{tabular}

not taken into account (they can be accounted for at the level of the function similarity calculation).

We assume given two similarity measures. $\sigma_{V}$ measures the similarity between two functions $i \in V_{A}$ and $i^{\prime} \in V_{B}$ such that $\sigma_{V}\left(i, i^{\prime}\right)=s_{i i^{\prime}} . \sigma_{E}$ measures the similarity between function calls. If $i$ calls $j$ in $A$ and $i^{\prime}$ calls $j^{\prime}$ in $B$, the similarity of those calls is $\sigma_{E}\left((i, j),\left(i^{\prime}, j^{\prime}\right)\right)=s_{i i^{\prime} j j^{\prime}}$. We assume that the similarities give values in $[0,1]$. This enables us to convert similarities into costs using $d_{i i^{\prime}}=1-s_{i i^{\prime}}$ and $d_{i i^{\prime} j j^{\prime}}=1-$ $s_{i i^{\prime} j j^{\prime}}$. Finally, we assume given two non-negative constant values $d_{\epsilon}$ and $d_{\epsilon \epsilon}$ corresponding to the cost of insertion or deletion of a function and call in a call graph.

We denote any series of graph edit operations $P=$ $\left(o p_{1}, \ldots, o p_{k}\right)$ an edit path, and define $\mathcal{P}(A, B)$ as the set of all possible edit paths that transform $G_{A}$ into $G_{B}$. Formally, if $\left(o p_{1}, \ldots, o p_{k}\right) \in \mathcal{P}(A, B)$, then $o p_{k}\left(o p_{k-1}\left(\ldots o p_{1}\left(G_{A}\right) \ldots\right)\right)=G_{B}$. We finally denote $C(P)=\sum_{i=1}^{k} c\left(o p_{i}\right)$ the cost the edit path $P$.

Table I lists the six possible graph edit operations we consider, with their respective costs. In this paper, we restrict $\mathcal{P}(A, B)$ to the set of restricted edit paths [25]. An important property of such paths is that they correspond to a unique mapping between the functions of $A$ and those of $B$ (see the Appendix for details). Note that unlike common GED definitions, our formulation implies a constant cost for every function (or call) insertion or deletion, whatever its content.

Based on these definitions, our formulation of the binary diffing problem consists in finding the minimal-cost edit path $P^{*}$ that transforms A into B. Formally:

$$
\begin{aligned}
P^{*} & =\underset{P \in \mathcal{P}(A, B)}{\arg \min } C(P), \\
& =\underset{\left(o p_{1}, \ldots, o p_{k}\right) \in \mathcal{P}(A, B)}{\arg \min } \sum_{i=1}^{k} c\left(o p_{i}\right) .
\end{aligned}
$$

\section{B. Binary diffing as a network alignment problem}

We now reformulate our definition of the binary diffing problem as an equivalent instance of a network alignment problem.

We first describe the diffing correspondences via a binary vector $\mathbf{x} \in\{0,1\}^{\left|V_{A}\right| \times\left|V_{B}\right|}$ (where $|U|$ denotes the cardinality of the set $U$ ) for which $x_{i i^{\prime}}=1$ if and only if function $i$ in $A$ is matched with function $i^{\prime}$ in $B$. To ensure that each function from $A$ is matched to at most one function in $B$ and vice versa, $\mathrm{x}$ must fulfil the following constraints:

$$
\forall i \in V_{A}, \sum_{j^{\prime} \in V_{B}} x_{i j^{\prime}} \leq 1, \quad \forall i^{\prime} \in V_{B}, \sum_{j \in V_{A}} x_{j i^{\prime}} \leq 1 .
$$

A good matching should associate similar functions that have also similar calling patterns. This can be captured in a cost matrix $Q \in \mathbb{R}^{\left|V_{A}\right|^{2} \times\left|V_{B}\right|^{2}}$ defined as follows:

$$
Q_{i i^{\prime} j j^{\prime}}= \begin{cases}w_{i i^{\prime}} & \text { if } i i^{\prime}=j j^{\prime}, \\ w_{i i^{\prime} j j^{\prime}} & \text { if }(i, j) \in E_{A} \text { and }\left(i^{\prime}, j^{\prime}\right) \in E_{B}, \\ 0, & \text { otherwise. }\end{cases}
$$

with

$$
w_{i i^{\prime}}=s_{i i^{\prime}}+2 d_{\epsilon}-1, \quad w_{i i^{\prime} j j^{\prime}}=s_{i i^{\prime} j j^{\prime}}+2 d_{\epsilon \epsilon}-1 .
$$

Using these definitions, it can be shown that computing the optimal edit path of GED is equivalent to solving the following network alignment problem:

$$
\begin{aligned}
\mathbf{x}^{*}=\underset{\mathbf{x}}{\arg \max } & \mathbf{x}^{T} Q \mathbf{x} \\
\text { subject to } & \forall i \in V_{A}, \sum_{j^{\prime} \in V_{B}} x_{i j^{\prime}} \leq 1 \\
& \forall i^{\prime} \in V_{B}, \sum_{j \in V_{A}} x_{j i^{\prime}} \leq 1
\end{aligned}
$$

We provide a proof in the Appendix.

\section{Graph edit operation costs}

1) Local vs global similarity trade-off: The definition of the edit operation costs of any GED formulation usually relies on carefully chosen data based considerations (see e.g. [4]). Costs have obviously an effect on the quality of the matching but also on the ability of a solver to find an approximately optimal solution. Moreover, because of the difficulty of function comparisons, local similarities might be inconsistent with the call patterns and there may be no solution optimal both locally and globally. Therefore, a matching results from an inherent tradeoff between local node similarity and global graph topology.

In order to control the trade-off one can decompose $Q$ into two terms and weight them. We define $Q_{1}$ as the diagonal matrix in $\mathbb{R}^{\left|V_{A}\right|^{2} \times\left|V_{B}\right|^{2}}$ with diagonal terms $Q_{1 i i^{\prime} i i^{\prime}}=w_{i i^{\prime}}$ and $Q_{2}$ as $Q_{2}=Q-Q_{1}$. $Q_{1}$ gathers the function/node similarities while $Q_{2}$ contains all the potential induced overlapping edges, called "squares". A potential square consists of a pair of edges in both call graphs: $(i, j) \in E_{A}$ and $\left(i^{\prime}, j^{\prime}\right) \in E_{B}$. If $i$ is matched to $i^{\prime}$ and $j$ to $j^{\prime}$, then the call structure is preserved and, in a sense, forms a square (with two sides coming from the matches and two sides coming from the calls).

Given a trade-off parameter $\alpha \in[0,1]$, the objective function of (NAP can thus be modified into:

$$
\alpha \mathbf{x}^{T} Q_{1} \mathbf{x}+(1-\alpha) \mathbf{x}^{T} Q_{2} \mathbf{x} .
$$

In terms of graph edit operations, this reformulation consists in appropriately weighting the original edit operation costs.

Notice that extreme cases for $\alpha$ correspond to some interesting particular cases. When $\alpha=1$, we recover a maximum weight matching (MWM) strategy which disregards the calls while $\alpha=0$ corresponds to a maximum common edge subgraph instance (MCS) where function similarities are not used. Therefore, our formulation can be seen as a balanced strategy between the two most common binary code matching methods. 
TABLE II: Function features and respective weights used in our proposed similarity measure. The final similarity score is computed using the Canberra distance.

\begin{tabular}{lcl}
\hline Type & Weight & Features \\
\hline Content & 23 & $\begin{array}{l}\text { total \# of instructions } \\
\text { \# of instructions per class } \\
\text { max \# of block instructions }\end{array}$ \\
\hline Topology & 19 & $\begin{array}{l}\text { \# of blocks } \\
\text { max jumps } \\
\text { max of block callers } \\
\text { max \# of block callees }\end{array}$ \\
\hline Neighboorhood & 7 & $\begin{array}{l}\text { \# of function callers } \\
\text { \# of function callees }\end{array}$ \\
\hline
\end{tabular}

2) Function content similarity: In this paper, we propose a simple function similarity metric $\sigma_{V}$. It consists in a weighted Canberra distance [26] over the set of features given in Table III. During the computation, each feature is properly weighted according to its type. We distinguish content based (instructions), topological based (CFG layout), and neighborhood based features (CG callers and callees). Note that one of our feature refers to an instruction classification. This classification encodes each instruction using the class of its mnemonic and the ones of its potential operands. Our taxonomy consists in respectively 34 and 13 different mnemonic and operand classes.

Since most matching algorithms are sensitive to ties between function distances, we introduce a small perturbation to the resulting similarity scores. Assuming that the denomination of the functions is consistent with their order in terms of entry address, the similarity between function $i$ in $A$ and $i^{\prime}$ in $B$ is being increased by the value $1-\frac{\left|i-i^{\prime}\right|}{\max \left(\left|V_{A}\right|,\left|V_{B}\right|\right)}$.

3) Function call similarity: In order to measure the similarity of two function calls, we simply use a $0 / 1$ indicator, i.e. $\sigma_{E}\left((i, j),\left(i^{\prime}, j^{\prime}\right)\right)=1$ if and only if $(i, j) \in E_{A}$ and $\left(i^{\prime}, j^{\prime}\right) \in E_{B}$. Therefore, the matrix $Q_{2}$ can be computed through the Kronecker product of the affinity matrix of $G_{A}$ and $G_{B}$.

Finally, in order to compare with other state of the art methods, and because, in general, binary diffing favors recall over precision, we set the insertion/deletion operation costs to $d \epsilon=d_{\epsilon \epsilon}=\frac{1}{2}$. This forces the algorithm to produce a complete mapping even when some assignments are of poor relevance.

As pointed out in Section II-B, other similarity measures have been proposed, some of them being much more sophisticated than the one we propose to use. However, this paper aims at identifying the benefit of the proposed matching approach and as such a simple metric seems more appropriate to emphasize the effect of variations in matching.

\section{NETWORK ALIGNMENT WITH MAX PRODUCT BELIEF PROPAGATION}

The network alignment problem, sometimes also referred to as graph matching problem, is an important optimization problem that has been extensively studied for decades [27]. Although it is not easier to solve the NAP than the GED, several efficient approximate algorithms have been proposed, based on spectral methods [28], [29], convex or indefinite relaxations [30], [31] or linearization [32].

In this section, we introduce a novel algorithm to efficiently approximate the binary diffing problem as a network alignment problem. This algorithm is inspired of a previous model of [5], which proposes to address the integer program (NAP) through an equivalent graphical model mode inference.

A graphical model is a way to represent a class of probability distributions over some random variables [33]. There is a strong link between inference in graphical models and optimization, especially when we consider Maximum A Posteriori (MAP) inference. This is the general problem of finding the most probable value of some of the random variables given the value of the rest of the variables. A particular case of MAP inference is to find the mode of a probability distribution, i.e. the most probable value of all its variables. One of the most efficient algorithm for MAP inference is the max-product algorithm. It consists in passing messages between the vertices of the graphical model that represents the distribution (see e.g. [33], chapter 13).

To leverage this algorithm, we design a graphical model that encodes both the objective function and the constraints of NAP into a probability distribution such that it assigns maximum probability to the optimal assignment of the NAP. Finding the mode of the distribution is then equivalent to solving the NAP.

Formally, matching vector $x$ is associated to binary random variables $X=\left\{X_{i i^{\prime}} \in\{0,1\}, i i^{\prime} \in V_{A} \times V_{B}\right\}$. The constraints (1) of NAP are encoded through Dirac measures $f_{i}:\{0,1\}^{\left|\partial \overrightarrow{f_{i}}\right|} \rightarrow\{0,1\}$ and $g_{i^{\prime}}:\{0,1\}^{\left|\partial g_{i^{\prime}}\right|} \rightarrow\{0,1\}$ such that:

$$
\begin{gathered}
\forall i \in V_{A}, f_{i}\left(x_{\partial f_{i}}\right)= \begin{cases}1, & \text { if } \sum_{j^{\prime} \in V_{B}} x_{i j^{\prime}} \leq 1, \\
0, & \text { otherwise. }\end{cases} \\
\forall i^{\prime} \in V_{B}, g_{i^{\prime}}\left(x_{\partial g_{i^{\prime}}}\right)= \begin{cases}1, & \text { if } \sum_{j \in V_{A}} x_{j i^{\prime}} \leq 1, \\
0, & \text { otherwise, }\end{cases}
\end{gathered}
$$

where $x_{\partial f_{i}}=\left\{x_{i j^{\prime}} \in \mathbf{x}, j^{\prime} \in V_{B}\right\}$, and similarly for $x_{\partial g_{i^{\prime}}}$.

The probability distribution of the corresponding graphical model is then:

$$
p_{X}(\mathbf{x})=\frac{1}{Z}\left[\prod_{i=1}^{n} f_{i}\left(x_{\partial f_{i}}\right) \prod_{i^{\prime}=1^{\prime}}^{m^{\prime}} g_{i^{\prime}}\left(x_{\partial g_{i^{\prime}}}\right)\right] e^{\mathbf{x}^{T} Q \mathbf{x}}
$$

Where the normalization constant $Z$ denotes the partition function of the model.

It is clear that the support of the distribution (2) is equivalent to the set of feasible solutions in $(\overline{\mathrm{NAP}})$. Furthermore, the mode of $p_{X}(\mathbf{x})$ corresponds to the optimal solution of (NAP).

In our work, we introduce modifications to the original model of [5] in order to speed up the computation and favor the messages convergence. Though the details and improvements of these modifications are out of scope for this paper, we provide a complete derivation of the message passing framework in the Appendix.

A key property of this model is the local structure of the message passing scheme. This later limits the propagation 
of updates to the connected components only, and therefore reduces the overall computation cost of the problem when working on sparse graphs, which is generally the case of call graphs. Moreover, it enables to discard some potential correspondences considered too unlikely, and thus significantly reduce the size of the problem solution set. This property is very useful to control the required computation cost and memory usage of larger problem instance: as shown in e.g. [34], the cost of one iteration of our algorithm is in $O\left(n n z\left(Q_{1}\right)+\right.$ $n n z\left(Q_{2}\right)$ ) where $n n z(x)$ denotes the number of non-zero entries in $x$. Note that after the last iteration, we need to solve a MWM problem which adds a $O\left(n n z\left(Q_{1}\right) N+N^{2} \log N\right)$ cost to the whole procedure, where $N=\left|V_{A}\right|+\left|V_{B}\right|$.

Our implementation includes a sparsity ratio parameter $\xi \in[0,1]$ in order to remove a ratio of less probable correspondences and forces the algorithm to find a solution among the remaining ones.

\section{Evaluation}

This section is dedicated to a thorough evaluation of our proposed solution, named QBinDiff, and to a comparison of its performances with a selection of state-of-art diffing approaches. We describe first our evaluation benchmark, then the chosen binary code similarity and finally the experiments.

\section{A. Benchmark}

A diffing approach can be evaluated by comparing the mapping results with "true" assignments, known as the ground truth. Unfortunately, such assignments are not readily available and may be in fact very difficult to determine in an objective way. As part of this work, we have built a new benchmark that will be released to the research community.

1) Benchmark design: To select programs to include in the proposed benchmark, we have considered several requirements. First, the source of the programs should be made readily available, within several different versions. This enables us to compile the program with symbols and thus ease the determination of the ground truth. Second, well maintained source repositories with explicit commit descriptions, detailed change $\operatorname{logs}$, as well as a relatively consistent function denomination over time are also very important features for the ground truth extraction. Third, as this extraction is largely done manually, program sizes should be "reasonable".

According to these considerations, we choose three well known open source project to compose our experimental dataset, namely $\mathrm{Zlib}^{1}$, Libsodium ${ }^{2}$ and OpenSSL $4^{3}$ Note that some of these programs are amongst the most frequently used for evaluation in the literature [1].

For each of these projects, we first downloaded the official repository, then we compiled the different available versions using GCC v7.5 for x86-64 target architecture with -O3 optimization level and keeping the symbols. Once extracted, each binary was stripped to remove all symbols, then disassembled

\footnotetext{
${ }^{1}$ https://github.com/madler/zlib

${ }^{2}$ https://github.com/jedisct1/libsodium

${ }^{3}$ https://github.com/openssl/openssl
}

TABLE III: Description of our binary diffing dataset. The last six columns respectively record the number of different binary versions, the number of resulting diffing instances, the average number of functions and function calls and the average ratio of conserved functions in our manually extracted and extrapolated ground truth.

\begin{tabular}{lrrrrrr}
\hline Program & Vers. & Diff. & Nodes & Edges & GT & $\overline{\text { GT }}$ \\
\hline Zlib & 18 & 153 & 153 & 235 & 0.99 & 0.96 \\
Libsodium & 33 & 528 & 589 & 701 & 0.98 & 0.79 \\
OpenSSL & 17 & 136 & 3473 & 18563 & 0.94 & 0.72 \\
\hline
\end{tabular}

using IDA Pro v7.2 $2^{4}$ and finally exported into a readable file with the help of BinExpor 5 During the problem statement, only plain text functions determined during the disassembly process are considered.

This extraction protocol provided us with respectively 18 , 33 and 17 different binary versions. For each project, given $n$ different versions of the program, we propose to evaluate our method in diffing all the $\frac{n(n-1)}{2}$ possible pairs of different executables. Statistics describing our evaluation dataset are given in Table III

Notice that both the average call graph size and density of the programs varies with the different projects. This variety will provide insights on the scalability of the diffing methods under study as well as the effect of sparsity on our solver.

2) Ground Truth: As recalled in Section III determining the 'true assignment' between the functions of two given binaries is a difficult task. In its strongest sense, this problem reduces to evaluate the semantic equivalence between two pieces of code and is known to be undecidable [1].

In practice, when working on different versions of the same, open-source and well documented binary, one may significantly reduce the difficulty by carefully exploiting the human readable information available in both the source code and the binary symbols. Moreover, some project repositories include detailed commit descriptions that precisely record the modification from a version to another. However, these information almost always refer to the changes occurring during a release, and are thus only available for contiguous versions. Therefore, in order to obtain the function assignment among two arbitrary program versions, one must extrapolate the different mappings of the releases that happened in the meantime.

Our ground truth extraction protocol has two steps. We first manually determine what we think to be the function mapping that best describes the modifications between two successive binary versions. This process is done with regards to the Changelogs files, the source code and the unstripped binaries. Excepted for few major project modifications, almost all the functions are mapped from a version to its successor (see Table III).

Once all the contiguous version ground truth mappings are extracted, we deduce all the pairwise diffing correspondences

\footnotetext{
${ }^{4}$ https://www.hex-rays.com/products/ida

${ }^{5}$ https://github.com/google/binexport
} 
by extrapolating the mappings from version to versions. Formally, if we encode the mapping between $A_{1}$ and $A_{2}$ into a boolean matrix $M_{A_{1} \rightarrow A_{2}}$ such that $M_{A_{1} \rightarrow A_{2} i i^{\prime}}=1$ if and only if function $i$ in $A_{1}$ is paired with function $i^{\prime}$ in $A_{2}$, then, our extrapolating scheme simply consists in computing the diffing correspondence between $A_{k}$ and $A_{n}$ as follows: $M_{A_{k} \rightarrow A_{n}}=\prod_{i=k}^{n-1} M_{A_{i} \rightarrow A_{i+1}}$.

\section{B. Experimental setup}

All the experiments have been conducted on an identical hardware 6 , using the implementation provided by the authors when possible.

Our method, QBinDiff, is used with its default parameters ( $\alpha=0.75$ and $\epsilon=0.5$ ), and within a maximum of 1000 iterations. We set the sparsity ratio parameter $\xi$ to 0 for smaller projects Zlib and Libsodium and to 0.9 for OpenSSL.

We compare our method with BinDiff [19], a closed source state-of-the-art binary diffing tool which uses a matching algorithm very close to MCS, but based on different, non public, function similarity heuristics.

In addition, our approach is compared to differs constructed by combinations chosen among three function similarity measures and two matching algorithms outlined in Section III All combinations are used. The baselines are described below.

1) Function content similarity: To evaluate the impact of the function similarity measure on the diffing process, three state-of-the-art binary code similarity approaches have been selected: Gemini [13], GraphMatching (GraphM.) [14] and DeepBinDiff (DeepBD.) [16].

Gemini and GraphMatching are supervised learning models that require to be trained on multiple pairs of functions labeled as similar or different. As the manual construction of such a dataset is tedious, existing methods usually use a collection of functions extracted from slightly mutated programs, such as different versions of an executable. Then, a pair of functions is labeled as similar if they share the same (or very similar) name, and dissimilar otherwise.

We applied this protocol to our dataset. Note that this should give a small competitive advantage to differs based on Gemini and GraphMatching as their similarity measures will be optimized on the specific type of functions found in the binaries under study.

During the training process, we collected 85680 samples of 7276 differently named functions from the unstripped binaries. $80 \%$ of them were used as training examples, $10 \%$ as a validation set and the remaining $10 \%$ were used to assess the final accuracy of the trained models. Both models were trained using their recommended hyper-parameters. To compute the similarity score of two embedded vectors, Gemini uses a cosine similarity measure, whereas GraphMatching refers to a normalized euclidean metric. After the training, the models respectively provided an estimated $\mathrm{AUC}^{7}$ of 0.968 and 0.939 .

We also trained DeepBinDiff instruction embedding model on each binary of our dataset, following the protocol and the

\footnotetext{
${ }^{6}$ Intel Xeon E5-2630 v4 @2.20GHz

${ }^{7}$ Area Under the ROC Curve
}

recommendations of the corresponding article [16]. As DeepBinDiff provides embeddings of basic blocks, we represent each function by the average of all its basic block embeddings.

2) Matching: Our matching algorithm is compared to the two most common methods found in the literature, namely MWM and MCS,

The MWM matching strategy implicitly used by Gemini and GraphMatching consists in solving the linear assignment problem based on the computed pairwise similarity scores. The exact solution of this problem can be found using conventional optimization solvers.

Several algorithms have been proposed to approximate the MCS problem. In order to compare with BinDiff and DeepBinDiff, we based our implementation on the one used in DeepBinDiff. However, since a CG is usually much more dense than a ICFG, we limited the neighbor parameter $k$ to 2. Note that, to output a complete mapping, the algorithm terminates by applying a MWM solver to the set of unmatched correspondences.

\section{Results}

The quality of a diffing result is measured using its precision and recall with respect to the ground truth. We refer to the standard definitions of precision and recall i.e. $p=\frac{|M \cap G|}{|G|}$ and $r=\frac{|M \cap G|}{|M|}$ where $M$ and $G$ respectively correspond to the set of matched function pairs in the computed and ground truth assignments. Note that, except for BinDiff, all the evaluated methods are designed to produce a complete mapping. In fact, none of them includes a mechanism to limit the mapping of the most unlikely correspondences during computation. Therefore, these matching strategies do not consider precision but only focuses on recall. In future work, we will investigate the effect of rising the insertion/deletion operation costs $d \epsilon$ and $d_{\epsilon \epsilon}$ in order to favor the solution's precision score.

Our experiments show that QBinDiff generally outperforms other matching approaches in both precision and recall (see Table IV, QBinDiff+NAP combinations). In fact, our method appears to perform clearly better at diffing more different programs, whereas it provides comparable solutions on similar binaries (see Figure 2). This highlights that the local greedy matching strategy of both MWM and MCS is able to provide good solutions on simple cases but generalizes poorly on more difficult problem instances. This results should be view as promising in the perspective of diffing much more different binaries.

Our NAP matching strategy can be applied with the state-ofthe-art function similarities chosen as reference. As observed with our custom metric, NAP provides better assignments than other matching approaches. Moreover, it appears that in almost all cases, the chosen matching strategy has more influence than the similarity metric. More surprisingly, the use of these complex models does not improve the accuracy of the resulting mapping, and might even worsen it in some case. Since the topology of the graphs does not change, this means that the computed similarity scores are not consistent with the actual ground truth assignment. In fact, it appears that both Gemini 


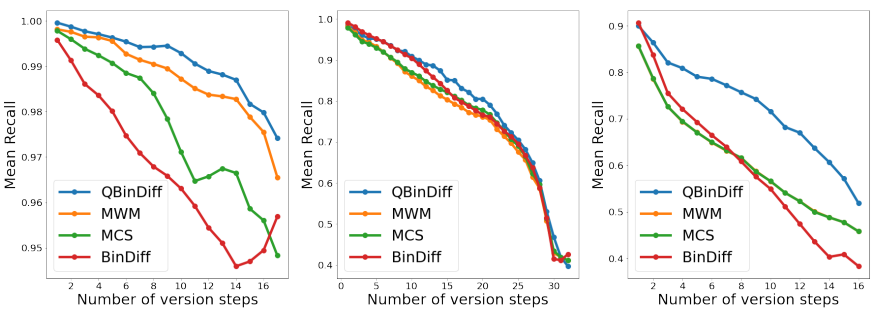

Fig. 2: Average recall scores according to the program versions distance. Every matching method provides comparable nearoptimal results while diffing very similar programs. As the distance increases, the performances of local matching strategies decline faster than our global approach.

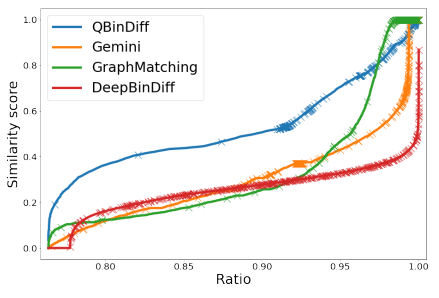

(a) Pairwise similarity scores

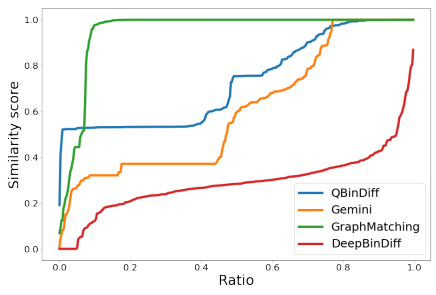

(b) Ground truth similarity scores
Fig. 3: Cumulative distribution function of all non-zero pairwise similarity scores (a) and of the ground truth pairs only (b) (libsodium-0.4.2 vs libsodium-1.0.3). The similarity scores in (a) that correspond to a ground truth correspondence are marked by a cross. GraphMatching appears to be well fitted to retrieve a large part of the correct matches but strongly deteriorates the score of some. QBinDiff provides a more balanced score but keeps almost all ground truth correspondence to a satisfying level.

and GraphMatching models very accurately retrieve similar functions, but strongly deteriorate the similarity scores of more diverging ones (see Figure 3). This is consistent with the original purpose of both model and with the training dataset which labels as completely different two similar functions with different names. In the case of DeepBinDiff, it seems that the scores of ground truth correspondences are distributed relatively uniformly over the cumulative distribution function, which means that the model itself does not provide sufficiently discriminative scores, and thus leads to erroneous assignments. Moreover, we were not able to compute DeepBinDiff embeddings on OpenSSL binaries in reasonable time. Indeed, these problem instances involve the factorization of the adjacency matrices of graphs of over 100000 nodes which is a very computationally intensive task.

An interesting analysis consists in comparing the different matching method assignments to the ground truth correspondences in terms of function similarity score and call graph alignment (see Figure 4). It appears that both Zlib and OpenSSL ground truth assignments are near-optimal in both maximum weight matching and maximum common edge subgraph scores. This observation is consistent with our exper-
TABLE IV: Average precision and recall scores for each combination of similarity measure (Similarity) and matching method (Matcher). The three tables correspond to the results on Zlib (top), Libsodium (middle) and OpenSSL (bottom) programs. The last two columns correspond to the similarity calculation time (Sim. calc.) and to the matching time (Time), both given in second.

\begin{tabular}{|c|c|c|c|c|c|}
\hline Similarity & Matcher & Precision & Recall & Sim. calc. & Time \\
\hline \multirow{3}{*}{ QBinDiff } & NAP & 0.955 & 0.995 & \multirow{3}{*}{3.0} & 0.2 \\
\hline & MWM & 0.953 & 0.992 & & 0.0 \\
\hline & MCS & 0.946 & 0.985 & & 0.0 \\
\hline \multirow{3}{*}{ Gemini } & NAP & 0.953 & 0.992 & \multirow{3}{*}{5.9} & 0.3 \\
\hline & MWM & 0.936 & 0.974 & & 0.0 \\
\hline & MCS & 0.942 & 0.981 & & 0.0 \\
\hline \multirow{3}{*}{ GraphM. } & NAP & 0.938 & 0.977 & \multirow{3}{*}{77.1} & 0.8 \\
\hline & MWM & 0.901 & 0.937 & & 0.0 \\
\hline & MCS & 0.927 & 0.964 & & 0.0 \\
\hline \multirow{3}{*}{ DeepBD. } & NAP & 0.909 & 0.946 & \multirow{3}{*}{489.3} & 4.7 \\
\hline & MWM & 0.820 & 0.853 & & 0.1 \\
\hline & MCS & 0.834 & 0.868 & & 0.2 \\
\hline BinDiff & BinDiff & 0.943 & 0.975 & 0.3 & 0.9 \\
\hline Similarity & Matcher & Precision & Recall & Sim. calc. & Matching \\
\hline \multirow{3}{*}{ QBinDiff } & NAP & 0.722 & 0.880 & \multirow{3}{*}{13.3} & 6.6 \\
\hline & MWM & 0.699 & 0.847 & & 0.2 \\
\hline & MCS & 0.704 & 0.854 & & 0.3 \\
\hline \multirow{3}{*}{ Gemini } & NAP & 0.714 & 0.863 & \multirow{3}{*}{24.2} & 7.5 \\
\hline & MWM & 0.668 & 0.802 & & 0.2 \\
\hline & MCS & 0.686 & 0.823 & & 0.2 \\
\hline \multirow{3}{*}{ GraphM. } & NAP & 0.693 & 0.837 & \multirow{3}{*}{293.9} & 21.7 \\
\hline & MWM & 0.643 & 0.776 & & 0.1 \\
\hline & MCS & 0.670 & 0.806 & & 0.3 \\
\hline \multirow{3}{*}{ DeepBD. } & NAP & 0.664 & 0.796 & \multirow{3}{*}{156.0} & 38.6 \\
\hline & MWM & 0.585 & 0.702 & & 1.9 \\
\hline & MCS & 0.599 & 0.718 & & 1.6 \\
\hline BinDiff & BinDiff & 0.752 & 0.869 & 0.4 & 0.9 \\
\hline Similarity & Matcher & Precision & Recall & Sim. calc. & Matching \\
\hline \multirow{3}{*}{ QBinDiff } & NAP & 0.605 & 0.783 & \multirow{3}{*}{88.6} & 213.3 \\
\hline & MWM & 0.522 & 0.670 & & 25.5 \\
\hline & MCS & 0.522 & 0.670 & & 24.4 \\
\hline \multirow{3}{*}{ Gemini } & NAP & 0.577 & 0.685 & \multirow{3}{*}{164.8} & 449.2 \\
\hline & MWM & 0.400 & 0.467 & & 24.9 \\
\hline & MCS & 0.401 & 0.467 & & 24.4 \\
\hline \multirow{3}{*}{ GraphM. } & NAP & 0.548 & 0.686 & \multirow{3}{*}{36999.2} & 2187.5 \\
\hline & MWM & 0.316 & 0.408 & & 54.7 \\
\hline & MCS & 0.317 & 0.409 & & 55.1 \\
\hline BinDiff & BinDiff & 0.572 & 0.681 & 0.7 & 3.2 \\
\hline
\end{tabular}

imental results that shows that a balanced network alignment matching strategy provide better accuracy results than other approaches. More importantly, it justifies our intuition that the proposed problem formulation as a network alignment problem is very well suited to address the binary diffing problem. However, in some cases, Libsodium correct assignments show to be sub-optimal in both function similarity and graph topology. In these cases, the ground truth mappings are inconsistent in both function content syntax and invoked call procedures. We investigated these cases, and noticed that over the versions, several functions were split in two such that a first trivial function is solely designed to access a second core function actually containing the whole function semantic. As we largely determined our ground truth based on function names, we mapped full functions into their newly created 

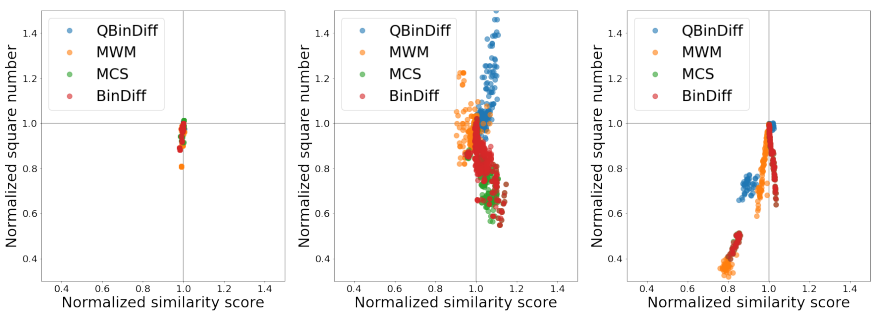

Fig. 4: Relative similarity scores and square numbers of different matching methods compared to the optimal assignment. The grey lines record the normalized scores of the ground truth. For Zlib (left) and OpenSSL (right) binaries, the ground truth seems to be a near optimal NAP assignment in almost all cases. This result does not hold for Libsodium (center), as in some cases, assignments computed by QBinDiff are better in both function similarity and number of induced squares.

accessors. We discuss these specific cases in the next section.

Our experiments also suggest that our rather basic function similarity metric provides scores that are consistent with the actual ground truth assignment (see Figure 4). Moreover, on the contrary to supervised learning models Gemini and GraphMatching, it produces less discriminative scores. Though it might be view as a less informative metric, it appears that this keeps the ground truth correspondences similarity scores at a satisfying level and ultimately results in better solutions (see Figure 3 .

Finally, we recorded the computing time of each methods. As could be expected, it takes much more time to approximate the NAP of two graphs than to compute the MWM or the MCS. However, this can be controlled by raising the sparsity ratio parameter $\xi$, at the cost of limiting the problem solution set and potentially resulting in sub-optimal assignments. Moreover, it seems that better similarity scores speed-up the computation. This is due to the fact that the algorithm finds more easily a satisfying local optima. Regarding the processing times, it appears that, whereas the use of Gemini model does not harm the required time, both GraphMatching and DeepBinDiff take very long time to compute the pairwise similarity scores, which might be prohibitive for larger programs.

\section{Limitations}

While it improves the state-of-the-art, our method could be further enhanced.

A first limitation is that our approach is designed to find a one-to-one correspondence between the functions of both programs. Thus, it can not properly handle cases where a function in a binary is split into several ones in the other program, or similarly, multiple functions are merged into a single one. In such case, the information of both the function syntax and its call graph relations is diluted into multiple chunks and may be harder to retrieve. Note that, to our knowledge, this problem is common to all other diffing methods, and that in practice, many function splits result in a core function containing most of the semantic information, and few trivial functions that are immediately called before or after it (as in the Libsodium programs). Such schemes could be handled by a pre-processing step.

The other key property of our approach is that it is based on the assumption that the true expected mapping is the optimal solution to the graph edit distance problem. Although it is partially validated by our limited sized experiments, there is no general available result that proves that this intuition is verified in practice, especially for more complex cross-compiler or cross-architecture diffing instances. One may argue that this only depends on the given graph edit operation costs definition. However, in practice, there is no known function similarity metric that exactly encodes the functions semantic and the interest of our method mostly rely on programs with rather similar call graphs. Moreover, the trade-off parameter $\alpha$ that balances the node and edge edit costs should be chosen carefully, which requires human expertise and prior knowledge about the binaries under analysis.

Finally, an important drawback of our problem formulation is that it requires a quartic memory matrix $Q$. Though we proposed to significantly reduce the problem size by limiting the solution set to the most probable correspondences, this relaxation inevitably induces information loss, especially for large graphs where the relaxation must rise consequently. In practice, binaries of several thousands of functions can be handled efficiently. For larger programs, it might be better to first partition the call graphs into smaller consistent subgraphs, and then proceed the matching among them. Although this partition is not trivial and might result in important diffing errors, it can be quite natural in modern programs for example following its modules.

\section{E. Threats to validity}

1) Internal validity: Our evaluation relies on a collection of diffing instances for which the ground truth assignment has been manually determined. Though we performed this extraction with regards to multiple sources of information such as source code, commit descriptions and unstripped symbols, we can not guarantee that our judgment is not biased, nor that it actually meets other experts opinion. Furthermore, any error or absence in our extracted mappings is later propagated in our extrapolation step. This may lower the confidence in the ground truth assignment between two distant versions. This threat is inherent to any manually determined assignments and can only be mitigated by releasing the dataset for the community to review.

2) External validity: Despite the relatively large number of proposed diffing instances, several factors still threaten the generalizability of our experiments. First, our benchmark only includes $\mathrm{C}$ programs taken from three open source projects. This is not representative of the variety of existing binaries. Moreover, all the executables were compiled with the same compiler, optimization level and targeted architecture. In future works, we will investigate the performance of our approach on programs built under different settings. Notice that this would probably require more sophisticated measures of similarity, 
able to efficiently handle greater syntactic differences. Last but not least, all our diffing instances compare different versions of a same program. Though the manual determination of an unanimous ground truth assignment between two different programs appears to be quite challenging, the evaluation of our method on such instances could be very instructive in the perspective of many applications such as the detection of vulnerability or of duplicate code.

3) Construct validity: The proposed comparison of our approach with other state of the art methods could also include threats to construct validity. First, all these methods are based on machine learning models that require a prior training step. We trained the models on the same dataset than the one we ran our experiments on. This could bias the resulting similarity scores, especially in case of overfitting. Moreover, we configured all models with their default parameters (recommended by the authors), though different settings could have provided better results. Finally, we must recall that none of the competitor methods where originally designed to address the exact same problem as ours. Indeed, both Gemini and GraphMatching have been initially proposed to retrieve nearduplicate functions whereas DeepBinDiff addresses the binary diffing problem at a basic bloc granularity.

\section{CONCLUSION}

In this paper, we introduced a new approach to address the binary diffing problem. It is based on its reformulation as a graph edit distance problem. This problem was shown to be equivalent to the network alignment problem, for which we derived an approximate message-passing algorithm. We proposed a new benchmark including hundreds of diffing ground truths and used it to compare the proposed approach to state of the art binary diffing methods.

Our experiments showed that our algorithm outperforms other existing approaches in almost every problem instances. It also highlighted that the matching strategy is a crucial part of the diffing process and has more influence than the choice of the function similarity measure. Moreover, it appeared that using similarity metrics originally designed to retrieve near duplicate functions might actually harm the quality of the resulting mapping. Finally, our results suggest that our problem formulation is a very adapted way to address the binary diffing problem.

Besides our formulation is quite natural and showed to result in more accurate mappings, it also provides a proper metric for measuring program-wide similarity. Indeed, any diffing assignment induces the (approximated) graph edit distance between the two programs. Therefore, our approach could also be used in a variety of metric-based analysis at a program level, such as library retrieval, program lineage, etc.

Finally, we believe that our graph matching algorithm could also be leveraged to perform diffing between matched functions in a post-processing step. This would results in a fined grained alignment between constitutive basic blocks of both functions and could provide to an analyst precious information about their exact differences.

\section{APPENDIX}

\section{Proof of equivalence of (GED) and (NAP)}

In the following proof, we first show that the solution set of (GED) can be reduced to the one of (NAP). Then we show that both objective functions are equivalent up to a sign and a constant term, which completes the proof.

Let $\mathcal{P}(A, B)$ be the set of all restricted edit path transforming $G_{A}$ into $G_{B}$ [25]. This set consists in edit paths where any node can be removed only if its incident edges have been previously removed, and where any edge can be inserted only if its terminal nodes previously existed or have been inserted. Moreover, no nodes or edges can be successively inserted then edited, edited then deleted, inserted then deleted, or edited multiple times. Finally, overlapping edge must be considered as an edition and can not result from a deletion then an insertion.

It can be shown [25] that any edit path in $P \in \mathcal{P}(A, B)$ can be fully characterized by a unique injective function of a subset $\hat{V}_{A}$ of $V_{A}$ to $V_{B}$, and reciprocally. Such a mapping can be encoded as a binary vector $\mathbf{x} \in\{0,1\}^{\left|V_{A}\right| \times\left|V_{B}\right|}$ such that $x_{i i^{\prime}}=1$ if and only if $i \rightarrow i^{\prime} \in P$ and satisfying the following constraints:

$$
\forall i \in V_{A}, \sum_{j^{\prime} \in V_{B}} x_{i j^{\prime}} \leq 1, \quad \forall i^{\prime} \in V_{B}, \sum_{j \in V_{A}} x_{j i^{\prime}} \leq 1 .
$$

Indeed, the injection implies that any node $i^{\prime} \in V_{B}$ is the image to at most one node $i \in V_{A}$. Therefore, $\forall i^{\prime} \in$ $V_{B}, \sum_{j \in V_{A}} x_{j i^{\prime}} \leq 1$. Moreover, any node $i \in \hat{V}_{A}$ has a unique image in $V_{B}$, so $\sum_{j^{\prime} \in V_{B}} x_{i j^{\prime}}=1$, whereas any node $j \in V_{A} \backslash \hat{V}_{A}$ is not part of the injection and $\sum_{j^{\prime} \in V_{B}} x_{j j^{\prime}}=0$.

Reciprocally, the constrained boolean vector defines a oneto-one mapping between the subsets of nodes $\hat{V}_{A} \in V_{A}$ and $\hat{V}_{B} \in V_{B}$. Thus, it implies a unique injection between $\hat{V}_{A}$ and the whole set of functions $V_{B}$.

Therefore, there is a bijection between the solution set of (GED) and the one of (NAP).

Let us now evaluate the cost of any arbitrary edit path $P \in$ $\mathcal{P}(A, B)$. Recall that this cost is completely induced by the edit operation on the nodes.

We first describe $C_{V}(P)$, the cost of the node operations in $P$. We distinguish the different possible operations such that:

$$
\begin{aligned}
C_{V}(P)= & \sum_{i \rightarrow i^{\prime} \in P} c\left(i \rightarrow i^{\prime}\right) \\
& +\sum_{i \rightarrow \epsilon \in P} c(i \rightarrow \epsilon)+\sum_{\epsilon \rightarrow i^{\prime} \in P} c\left(\epsilon \rightarrow i^{\prime}\right) \\
= & \sum_{i \rightarrow i^{\prime} \in P} d_{i i^{\prime}}-2 d_{\epsilon} \\
& +\sum_{i \rightarrow \epsilon \in P} d_{\epsilon}+\sum_{i \rightarrow i^{\prime} \in P} d_{\epsilon}+\sum_{\epsilon \rightarrow i^{\prime} \in P} d_{\epsilon}+\sum_{i \rightarrow i^{\prime} \in P} d_{\epsilon} \\
= & \sum_{i \rightarrow i^{\prime} \in P} d_{i i^{\prime}}-2 d_{\epsilon}+\sum_{i \in V_{A}} d_{\epsilon}+\sum_{i^{\prime} \in V_{B}} d_{\epsilon} \\
= & \sum_{i \rightarrow i^{\prime} \in P} d_{i i^{\prime}}-2 d_{\epsilon}+\left|V_{A}\right| d_{\epsilon}+\left|V_{B}\right| d_{\epsilon} .
\end{aligned}
$$


In order to evaluate the cost of all the edges operations, we must consider the different possible configurations for pairs of nodes. But first, we must introduce the following notations: $\delta_{i j}^{A}=\left\{\begin{array}{ll}=1, & \text { if }(i, j) \in E_{A} \\ =0, & \text { otherwise. }\end{array}\right.$, and similarly for $\delta_{i^{\prime} j^{\prime}}^{B}$.

We may now evaluate $C_{E}(P)$ such that:

$$
\begin{aligned}
C_{E}(P)= & \sum_{i \rightarrow i^{\prime} \in P} \sum_{j \rightarrow j^{\prime} \in P}\left[d_{i i^{\prime} j j^{\prime}} \delta_{i j}^{A} \delta_{i^{\prime} j^{\prime}}^{B}\right. \\
& \left.+d_{\epsilon \epsilon} \delta_{i j}^{A}\left(1-\delta_{i^{\prime} j^{\prime}}^{B}\right)+d_{\epsilon \epsilon}\left(1-\delta_{i j}^{A}\right) \delta_{i^{\prime} j^{\prime}}^{B}\right] \\
& +\sum_{i \rightarrow i^{\prime} \in P} \sum_{j \rightarrow \epsilon \in P} d_{\epsilon \epsilon} \delta_{i j}^{A}+\sum_{i \rightarrow i^{\prime} \in P} \sum_{\epsilon \rightarrow j^{\prime} \in P} d_{\epsilon \epsilon} \delta_{i^{\prime} j^{\prime}}^{B} \\
& +\sum_{i \rightarrow \epsilon \in P} \sum_{j \rightarrow j^{\prime} \in P} d_{\epsilon \epsilon} \delta_{i j}^{A}+\sum_{\epsilon \rightarrow i^{\prime} \in P} \sum_{j \rightarrow j^{\prime} \in P} d_{\epsilon \epsilon} \delta_{i^{\prime} j^{\prime}}^{B} \\
& +\sum_{i \rightarrow \epsilon \in P} \sum_{j \rightarrow \epsilon \in P} d_{\epsilon \epsilon} \delta_{i j}^{A}+\sum_{\epsilon \rightarrow i^{\prime} \in P} \sum_{\epsilon \rightarrow j^{\prime} \in P} d_{\epsilon \epsilon} \delta_{i^{\prime} j^{\prime}}^{B} \\
= & \sum_{i \rightarrow i^{\prime} \in P} \sum_{j \rightarrow j^{\prime} \in P}\left(d_{i i^{\prime} j j^{\prime}}-2 d_{\epsilon \epsilon}\right) \delta_{i j}^{A} \delta_{i^{\prime} j^{\prime}}^{B} \\
& +\sum_{i \in V_{A}} \sum_{j \in V_{A}} d_{\epsilon \epsilon} \delta_{i j}^{A}+\sum_{i^{\prime} \in V_{B}} \sum_{j^{\prime} \in V_{B}} d_{\epsilon \epsilon} \delta_{i^{\prime} j^{\prime}}^{B} \\
= & \sum_{i \rightarrow i^{\prime} \in P} \sum_{j \rightarrow j^{\prime} \in P}\left(d_{i i^{\prime} j j^{\prime}}-2 d_{\epsilon \epsilon}\right) \delta_{i j}^{A} \delta_{i^{\prime} j^{\prime}}^{B} \\
& +\left|E_{A}\right| d_{\epsilon \epsilon}+\left|E_{B}\right| d_{\epsilon \epsilon} .
\end{aligned}
$$

Putting all together, and denoting $C\left(P_{0}\right)=\left|V_{A}\right| d_{\epsilon}+$ $\left|V_{B}\right| d_{\epsilon}+\left|E_{A}\right| d_{\epsilon \epsilon}+\left|E_{B}\right| d_{\epsilon \epsilon}$, the cost of any edit path $P$ is:

$$
\begin{aligned}
C(P)= & C_{V}(P)+C_{E}(P) \\
= & C\left(P_{0}\right)+\sum_{i \rightarrow i^{\prime} \in P} d_{i i^{\prime}}-2 d_{\epsilon} \\
& +\sum_{i \rightarrow i^{\prime} \in P} \sum_{j \rightarrow j^{\prime} \in P}\left(d_{i i^{\prime} j j^{\prime}}-2 d_{\epsilon \epsilon}\right) \delta_{i j}^{A} \delta_{i^{\prime} j^{\prime}}^{B} \\
= & C\left(P_{0}\right)+\sum_{i i^{\prime} \in V_{A} \times V_{B}} x_{i i^{\prime}}\left(d_{i i^{\prime}}-2 d_{\epsilon}\right) \\
+ & \sum_{i i^{\prime} \in V_{A} \times V_{B}} \sum_{j j^{\prime} \in V_{A} \times V_{B}} x_{i i^{\prime}}\left(d_{i i^{\prime} j j^{\prime}}-2 d_{\epsilon \epsilon}\right) \delta_{i j}^{A} \delta_{i^{\prime} j^{\prime}}^{B} x_{j j^{\prime}} \\
= & C\left(P_{0}\right)-\sum_{i i^{\prime} \in V_{A} \times V_{B}} x_{i i^{\prime}} w_{i i^{\prime}} \\
& -\sum_{i i^{\prime} \in V_{A} \times V_{B}} \sum_{j j^{\prime} \in V_{A} \times V_{B}} x_{i i^{\prime}} w_{i i^{\prime} j j^{\prime}} \delta_{i j}^{A} \delta_{i^{\prime} j^{\prime}}^{B} x_{j j^{\prime}} \\
= & C\left(P_{0}\right)-\sum_{i i^{\prime} \in V_{A} \times V_{B}} \sum_{j j^{\prime} \in V_{A} \times V_{B}} x_{i i^{\prime}} w_{i i^{\prime} j j^{\prime}} x_{j j^{\prime}} \\
= & C\left(P_{0}\right)-\mathbf{x}^{T} Q \mathbf{x},
\end{aligned}
$$

where we simply use the fact that $d_{i i^{\prime}}-1-2 d_{\epsilon}+1=-w_{i i^{\prime}}$ and similarly for $w_{i i^{\prime} j j^{\prime}}$.

Therefore, exploiting the one-to-one correspondence between an edit path $P$ and its boolean representation $\mathbf{x}$, we may turn the minimization of GED into a maximization problem and obtain (NAP).

\section{QBinDiff's message passing scheme}

We reproduce the messages derivation of [5], and introduce our modifications. For the sake of simplicity, we changed the message passing denomination of the original paper to best highlight their vectorial structure.

The messages $\mathbf{f}=\left\{f_{i i^{\prime}}, w_{i i^{\prime}} \neq 0\right\}, \mathbf{g}=\left\{g_{i i^{\prime}}, w_{i i^{\prime}} \neq 0\right\}$ and $\mathbf{h}=\left\{h_{i i^{\prime} j j^{\prime}}, w_{i i^{\prime}} \neq 0 \cap w_{j j^{\prime}} \neq 0\right\}$ are all initialized to 0 .

At each iteration, the algorithm computes the following updates:

$$
\begin{aligned}
f_{i i^{\prime}}^{(t+1)}=w_{i i^{\prime}} & -\left(\max _{k \neq i} g_{k i^{\prime}}^{(t)}\right)_{+}-\gamma_{i i^{\prime}}^{(t)} \\
& +\sum_{j j^{\prime}}\left[w_{j j^{\prime} i i^{\prime}}+h_{j j^{\prime} i i^{\prime}}^{(t)}\right]_{0}^{w_{j j^{\prime} i i^{\prime}}} \\
g_{i i^{\prime}}^{(t+1)}=w_{i i^{\prime}} & -\left(\max _{k^{\prime} \neq i^{\prime}} f_{i k^{\prime}}^{(t)}\right)_{+}-\phi_{i i^{\prime}}^{(t)} \\
& +\sum_{j j^{\prime}}\left[w_{j j^{\prime} i i^{\prime}}+h_{j j^{\prime} i i^{\prime}}^{(t)}\right]_{0}^{w_{j j^{\prime} i i^{\prime}}} \\
h_{i i^{\prime} j j^{\prime}}^{(t+1)}=w_{i i^{\prime}} & -\left(\max _{k^{\prime} \neq i^{\prime}} f_{i k^{\prime}}^{(t)}\right)_{+}-\phi_{i i^{\prime}}^{(t)} \\
& -\left(\max _{k \neq i} g_{k i^{\prime}}^{(t)}\right)_{+}-\gamma_{i i^{\prime}}^{(t)} \\
& +\sum_{k k^{\prime} \neq j j^{\prime}}\left[w_{k k^{\prime} i i^{\prime}}+h_{k k^{\prime} i i^{\prime}}^{(t)}\right]_{0}^{w_{k k^{\prime} i i^{\prime}}},
\end{aligned}
$$

where: $x_{+}=\max (0, x)$, and $[x]_{a}^{b}= \begin{cases}=a, & \text { if } x \leq a, \\ =x, & \text { if } a<x<b \\ =b, & \text { otherwise, }\end{cases}$ and where we introduced Bertsekas' $\epsilon$-complementary slackness mechanism [35]:

$$
\begin{aligned}
\phi_{i i^{\prime}}^{(t)} & = \begin{cases}\epsilon, & \text { if } f_{i i^{\prime}}^{(t)} \neq \max _{k^{\prime}} f_{i k^{\prime}}^{(t)}, \\
0, & \text { otherwise. }\end{cases} \\
\gamma_{i i^{\prime}}^{(t)} & = \begin{cases}\epsilon, & \text { if } g_{i i^{\prime}}^{(t)} \neq \max _{k} g_{k i^{\prime}}^{(t)}, \\
0, & \text { otherwise. }\end{cases}
\end{aligned}
$$

At the end of iteration $t$, the estimated mode is achieved by:

$$
\hat{x}_{i i^{\prime}}^{(t)}= \begin{cases}=1, & \text { if } \hat{p}_{X_{i i^{\prime}}}^{(t)}>0 \\ =0, & \text { otherwise }\end{cases}
$$

where $\hat{p}_{X_{i i^{\prime}}}^{(t)}$ denotes the log-ratio of the estimated marginal distribution of $x_{i i^{\prime}}$ :

$$
\begin{aligned}
\hat{p}_{X_{i i^{\prime}}}^{(t)}=w_{i i^{\prime}} & -\left(\max _{k^{\prime} \neq i^{\prime}} f_{i k^{\prime}}^{(t)}\right)_{+}-\phi_{i i^{\prime}}^{(t)} \\
& -\left(\max _{k \neq i} g_{k i^{\prime}}^{(t)}\right)_{+}-\gamma_{i i^{\prime}}^{(t)} \\
& +\sum_{j j^{\prime}}\left[w_{j j^{\prime} i i^{\prime}}+h_{j j^{\prime} i i^{\prime}}^{(t)}\right]_{0}^{w_{j j^{\prime} i i^{\prime}}}
\end{aligned}
$$




\section{REFERENCES}

[1] I. U. Haq and J. Caballero, "A Survey of Binary Code Similarity," ACM Computing Surveys, vol. 54, no. 3, pp. 51:1-51:38, Apr. 2021.

[2] X. Hu, T.-c. Chiueh, and K. G. Shin, "Large-scale malware indexing using function-call graphs," in Proceedings of the 16th ACM conference on Computer and communications security, ser. CCS '09. Chicago, Illinois, USA: Association for Computing Machinery, Nov. 2009, pp. 611-620.

[3] O. Kostakis, J. Kinable, H. Mahmoudi, and K. Mustonen, "Improved call graph comparison using simulated annealing," in Proceedings of the 2011 ACM Symposium on Applied Computing, ser. SAC '11. TaiChung, Taiwan: Association for Computing Machinery, Mar. 2011, pp. 15161523.

[4] M. Bourquin, A. King, and E. Robbins, "BinSlayer: accurate comparison of binary executables," in Proceedings of the 2nd ACM SIGPLAN Program Protection and Reverse Engineering Workshop, ser. PPREW '13. Rome, Italy: Association for Computing Machinery, Jan. 2013, pp. 1-10.

[5] M. Bayati, M. Gerritsen, D. F. Gleich, A. Saberi, and Y. Wang, "Algorithms for Large, Sparse Network Alignment Problems," in Proceedings of the 2009 Ninth IEEE International Conference on Data Mining, ser. ICDM '09. USA: IEEE Computer Society, Dec. 2009, pp. 705-710.

[6] X. Meng and B. P. Miller, "Binary code is not easy," in Proceedings of the 25th International Symposium on Software Testing and Analysis, ser. ISSTA 2016. New York, NY, USA: Association for Computing Machinery, Jul. 2016, pp. 24-35.

[7] L. Massarelli, G. A. Di Luna, F. Petroni, L. Querzoni, and R. Baldoni, "Investigating Graph Embedding Neural Networks with Unsupervised Features Extraction for Binary Analysis," in Proceedings 2019 Workshop on Binary Analysis Research. San Diego, CA: Internet Society, 2019.

[8] K. Redmond, L. Luo, and Q. Zeng, "A Cross-Architecture Instruction Embedding Model for Natural Language Processing-Inspired Binary Code Analysis," in Proceedings 2019 Workshop on Binary Analysis Research. San Diego, CA: Internet Society, 2019.

[9] X. Zhang, W. Sun, J. Pang, F. Liu, and Z. Ma, "Similarity Metric Method for Binary Basic Blocks of Cross-Instruction Set Architecture," in Proceedings 2020 Workshop on Binary Analysis Research. San Diego, CA: Internet Society, 2020.

[10] M. Cova, V. Felmetsger, G. Banks, and G. Vigna, "Static Detection of Vulnerabilities in x86 Executables," in Proceedings of the 22nd Annual Computer Security Applications Conference, ser. ACSAC '06. USA: IEEE Computer Society, Dec. 2006, pp. 269-278.

[11] D. Gao, M. K. Reiter, and D. Song, "BinHunt: Automatically Finding Semantic Differences in Binary Programs," in Proceedings of the 10th International Conference on Information and Communications Security, ser. ICICS '08. Berlin, Heidelberg: Springer-Verlag, Oct. 2008, pp. 238-255.

[12] Y. David, N. Partush, and E. Yahav, "Statistical similarity of binaries," in Proceedings of the 37th ACM SIGPLAN Conference on Programming Language Design and Implementation, ser. PLDI '16. Santa Barbara, CA, USA: Association for Computing Machinery, Jun. 2016, pp. 266 280.

[13] X. Xu, C. Liu, Q. Feng, H. Yin, L. Song, and D. Song, "Neural Networkbased Graph Embedding for Cross-Platform Binary Code Similarity Detection," in Proceedings of the 2017 ACM SIGSAC Conference on Computer and Communications Security, ser. CCS '17. Dallas, Texas, USA: Association for Computing Machinery, Oct. 2017, pp. 363-376.

[14] Y. Li, C. Gu, T. Dullien, O. Vinyals, and P. Kohli, "Graph Matching Networks for Learning the Similarity of Graph Structured Objects," in International Conference on Machine Learning. PMLR, May 2019, pp. 3835-3845.

[15] S. H. H. Ding, B. C. M. Fung, and P. Charland, "Asm2Vec: Boosting Static Representation Robustness for Binary Clone Search against Code Obfuscation and Compiler Optimization," in 2019 IEEE Symposium on Security and Privacy (SP), May 2019, pp. 472-489.

[16] Y. Duan, X. Li, J. Wang, and H. Yin, "DeepBinDiff: Learning ProgramWide Code Representations for Binary Diffing," in Proceedings 2020 Network and Distributed System Security Symposium. San Diego, CA: Internet Society, 2020.

[17] H. Gao and H. Huang, "Deep attributed network embedding," in Proceedings of the 27th International Joint Conference on Artificial Intelligence, ser. IJCAI'18. Stockholm, Sweden: AAAI Press, Jul. 2018, pp. 3364-3370.
[18] H. W. Kuhn, "The Hungarian method for the assignment problem," Naval Research Logistics Quarterly, vol. 2, no. 1-2, pp. 83-97, 1955.

[19] T. Dullien, "Graph-based comparison of executable Objects," SSTIC'05 : Symposium sur la Securite des Technologies de l'Information et des Communications, Rennes, France, June 2005, 2005.

[20] L. P. Cordella, P. Foggia, C. Sansone, and M. Vento, "A (Sub)Graph Isomorphism Algorithm for Matching Large Graphs," IEEE Transactions on Pattern Analysis and Machine Intelligence, vol. 26, no. 10, pp. 13671372, Oct. 2004.

[21] L. Bahiense, G. Manić, B. Piva, and C. C. De Souza, "The maximum common edge subgraph problem: A polyhedral investigation," Discrete Applied Mathematics, vol. 160, no. 18, pp. 2523-2541, Dec. 2012.

[22] K. Riesen, Structural Pattern Recognition with Graph Edit Distance: Approximation Algorithms and Applications, 1st ed. Springer Publishing Company, Incorporated, 2016.

[23] C.-L. Lin, "Hardness of Approximating Graph Transformation Problem," in Proceedings of the 5th International Symposium on Algorithms and Computation, ser. ISAAC '94. Berlin, Heidelberg: Springer-Verlag, Aug. 1994, pp. 74-82.

[24] K. Riesen and H. Bunke, "Approximate graph edit distance computation by means of bipartite graph matching," Image and Vision Computing, vol. 27 , no. 7 , pp. 950-959, Jun. 2009

[25] S. Bougleux, L. Brun, V. Carletti, P. Foggia, B. Gazre, and M. Vento, "Graph edit distance as a quadratic assignment problem," Pattern Recognition Letters, vol. 87, no. C, pp. 38-46, Feb. 2017.

[26] G. N. Lance and W. T. Williams, "Computer Programs for Hierarchical Polythetic Classification ("Similarity Analyses")," The Computer Journal, vol. 9, no. 1, pp. 60-64, May 1966.

[27] R. E. Burkard, E. Çela, P. M. Pardalos, and L. S. Pitsoulis, "The Quadratic Assignment Problem," in Handbook of Combinatorial Optimization: Volume1-3, D.-Z. Du and P. M. Pardalos, Eds. Boston, MA: Springer US, 1998, pp. 1713-1809.

[28] R. Singh, J. Xu, and B. Berger, "Global alignment of multiple protein interaction networks with application to functional orthology detection," Proceedings of the National Academy of Sciences, vol. 105, no. 35, pp. 12763-12 768, Sep. 2008.

[29] S. Zhang and H. Tong, "FINAL: Fast Attributed Network Alignment," in Proceedings of the 22nd ACM SIGKDD International Conference on Knowledge Discovery and Data Mining, ser. KDD '16. New York, NY, USA: Association for Computing Machinery, Aug. 2016, pp. 13451354.

[30] M. Zaslavskiy, F. Bach, and J.-P. Vert, "A Path Following Algorithm for the Graph Matching Problem," IEEE Transactions on Pattern Analysis and Machine Intelligence, vol. 31, no. 12, pp. 2227-2242, Dec. 2009.

[31] V. Lyzinski, D. E. Fishkind, M. Fiori, J. T. Vogelstein, C. E. Priebe, and G. Sapiro, "Graph Matching: Relax at Your Own Risk," IEEE Transactions on Pattern Analysis and Machine Intelligence, vol. 38, no. 1, pp. 60-73, Jan. 2016.

[32] G. W. Klau, "A new graph-based method for pairwise global network alignment," BMC Bioinformatics, vol. 10, no. 1, p. S59, Jan. 2009.

[33] D. Koller and N. Friedman, Probabilistic Graphical Models: Principles and Techniques - Adaptive Computation and Machine Learning. The MIT Press, 2009.

[34] A. M. Khan, D. F. Gleich, A. Pothen, and M. Halappanavar, "A multithreaded algorithm for network alignment via approximate matching," in Proceedings of the International Conference on High Performance Computing, Networking, Storage and Analysis, ser. SC '12. Washington, DC, USA: IEEE Computer Society Press, Nov. 2012, pp. 1-11.

[35] D. P. Bertsekas, "Auction algorithms for network flow problems: A tutorial introduction," Computational Optimization and Applications, vol. 1 , no. 1 , pp. 7-66, Oct. 1992 